\title{
On the Edge-balanced Index Set of a class of Power-cycle Nested Network Graph
}

\author{
Qingwen Zhang \\ Henan Polytechnic University, Jiaozuo, China \\ Email: zhangqingwenhua@163.com \\ Yuge Zheng \\ Henan Polytechnic University, Jiaozuo, China \\ Email: zhengyuge@hpu.edu.cn
}

\begin{abstract}
Based on the research of Power-cycle Nested Graph $C_{6^{m}} \times P_{m^{6}}$, the decomposition method of single point sector has come up. By the use of the process of clawed nested-cycle sub-graph, the edge-balanced index sets of the power-cycle nested graph $C_{6^{m}} \times P_{m^{6}}$ are solved when $m \geq 4$ and $m=4(\bmod 5)$. Besides, the constructive proofs of the computational formulas are also completed. The theory can be applied to information engineering, communication networks, computer science, economic management, medicine, etc. The proving method can be a reference to solve the problem of the power-cycle nested graph $C_{6^{m}} \times P_{m_{6}}$.
\end{abstract}

Index Terms -Edge-friendly labeling, edge-balanced index sets, power-cycle nested graph $C_{6^{m}} \times P_{m^{6}}$, clawed nested-cycle graph.

\section{INTRODUCTION}

\section{A. History}

In 1966 B.M.Stewart first introduced the theory of graph, by means of labeling function of vertex and edge. Since then, numerous domestic and international researchers have been working on the research of this aspect and winning a series of research results. In 1995, M.C.Kong and other scholars researched the concepts of the edge-balanced graph and the strong edge-balanced graph and put forward two conjectures. In 2002, B.L.C hen etc expanded edge-balance multiple graph concept in reference [2], proved that the conjecture in reference [1] is true and obtained a graph is edge-balanced is not NP-difficult problem. In 2006-2008, Alexander, Harris, etc made a research of vertex-balanced index sets and friendly index sets in reference [3-5]. In 2008, SubRyung used the structure method to study the new family of edge-balanced graph in reference [6]. In 2010, D Chopra researched the edge-balanced index sets of wheel graph in reference [7]. In 2011, Sin-Min Lee, C.C.Chou, M.Galiardi, M.K ong etc discussed the edgebalanced index sets of L-product of cycles with stars in reference [8]. Since 2011, Zheng Yuge and her students have researched the edge-balanced index sets of a class of chain graph in reference [9] and edge-balance index sets of the complete graphs reference [12]. Basing on this research, they also completely research the edgebalanced index sets of the equal-cycle nested graph in reference [13].

In graph of power-cycle nested graph $C_{6^{m}} \times P_{m^{6}}$, the difficulty of the problem multiplied when $n$ is bigger. In this article, by the use of the process clawed nestedcycle sub-graph and single point sector sub-graph, the edge-balanced index sets of the power-cycle nested graph $C_{6^{m}} \times P_{m^{6}}$ are solved when $m \geq 4$ and $m=4(\bmod 5)$.

\section{B. Definition}

A graph $G$ is an ordered pair $(V(G) ; E(G))$ consisting of a set $_{V(G)}$ of vertices and a set $E(G)$, disjoint from $_{V(G)}$, of edges, together with an incidence function that associates with each edge of $G$ an unordered pair of vertices of $G$. The graphs are all simple in this paper.

Definition 1.1 In graph $G$, let: $f: E(G) \rightarrow Z$ is a edge labeling function, namely to define $f\{e\}=0$ or 1 . The edge set labeling 0 or 1 is recorded as $E(0), E(1)$, using $e_{x}(0), e_{x}(1)$ to present the number of $E(0), E(1)$. According to the edge labeling $f$, we can define $f^{+}: V(G) \rightarrow\{0,1\}$, if $e_{x}(0)$ is more than $e_{x}(1)$,

$f^{+}(x)=0$, the vertex is labeling 0 ; if $e_{x}(0)$ is less than $_{e_{x}(1)}, f^{+}(x)=1$, the vertex is labeling 1 ; otherwise, the vertex $x$ is unlabeled. $e_{x}(0)$ or $e_{x}(1)$ presents the radix number of the edge collection that the edges linked $x$ are labeling 0 or 1 . The radix number of the vertex set $\mathrm{V}(0), \mathrm{V}(1)$ is presented by $v(0)$ or $v(1)$, respectively.

Definition 1.2 In graph $G$, let: $f: E(G) \rightarrow Z$ is an edge labeling function, if $|e(0)-e(1)| \leq 1, f$ is considered as an edge-friendly labeling of the graph $G$.

Definition 1.3 The edge-balanced index set of the graph $\operatorname{EBI}(G)$, is defined as $\{|v(0)-v(1)|$ : the edge labeling $\mathrm{f}$ is edge-friendly

Definition $1.4_{P_{m_{s}}}$ is a ray way that every road contains $m$ points, and that there are 6 branches at any points 
except the terminal point of each road.

Definition $1.5 C_{6^{m}}$ is a nested-cycle graph contains $m$ cycles, and recorded as the first circle, the second circle, $\cdots \cdots$ the $m$-th circle from inside to outside in turn. Among the i-th circle has $6^{i}$ vertex.

Definition 1.6 The power-cycle nested graph $C_{6^{m}} \times P_{m_{6}}$ is the Cartesian product of $C_{6^{m}}$ and $P_{m_{6}}$.

Two examples of graphs should serve to clarify the definition:
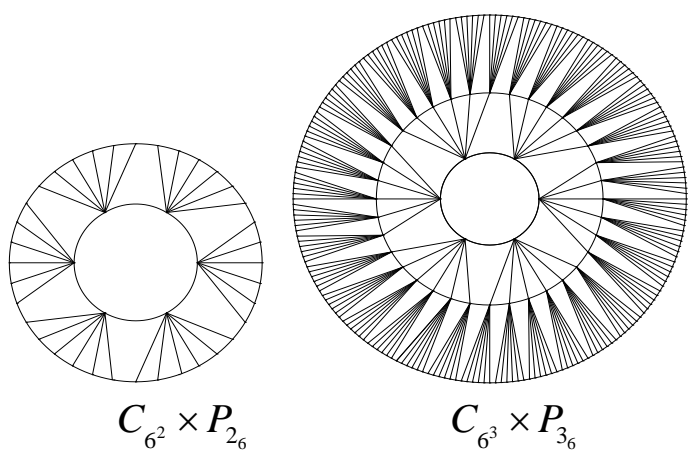

Figure 1

For clarity, the edge labeled $0(1)$ is named 0-edge (1edge), the vertex labeled $0(1)$ is named 0 -vertex (0vertex).

Simply, we will have the following marks: Recorded the $6^{i}$ vertices on the i-th cycle in clockwise order as: $i_{1}, i_{2}, i_{3}, \cdots, i_{6^{i}-1}, i_{6^{i}}$.The ray paths in the power-cycle nested graph are denoted by:

$$
\begin{gathered}
1_{i_{1}} \rightarrow 2_{i_{2}} \rightarrow 3_{i_{3}} \rightarrow \cdots \rightarrow(m-2)_{i_{(m-2)}} \rightarrow(m-1)_{i_{(m-1)}} \\
k \leq m) .
\end{gathered}
$$

Definition 1.7 In the power-cycle nested graph $C_{6^{m}} \times P_{m^{6}}$, a 1-vertex $X$ is considered to be saturated, if the $n$ edges linked to $X$ satisfy $e_{x}(1)=e_{x}(0)+2$ when the degree of vertex is even or the $n$ edges linked to $x$ satisfy $e_{x}(1)=e_{x}(0)+1$ when the degree of vertex is odd ; otherwise, the 1 -vertex $x$ is unsaturated. A 0 -vetex $x$ is considered to be saturated, if the n edges linked to $X$ are all 0 -edges; otherwise, the 0 -vertex $X$ is unsaturated.

Definition 1.8 In the power-cycle nested graph $C_{6^{m}} \times P_{m^{6}}$, let $m=5 t+4(t \in N)$,the induced sub-graph of the vertices, which the ray paths through the points with the vertices on the $5 t+4$ cycle as starting points and the vertices on the $5(t+1)+4$ cycle as the terminal points, denoted by $V_{t}^{\prime}$. And the graph $V_{t}^{\prime}$ subtract the edge on $5 t+2$ is thought as the clawed nested-cycle subgraph $V_{t}$.

In short, we introduce the concept of divisibility for power-cycle nested graph, it is obvious that
$C_{6^{\mathrm{m}}} \times P_{m_{6}}=C_{6^{2}} \times P_{2_{6}} \cup\left(\bigcup_{i=0}^{\frac{m-4}{5}} V_{i}\right)$, labeled the nested-cycle subgraph $V_{t}(t \in N)$. Based on the starting points of the ray paths, $V_{t}$ is divided into $6^{5 t+4}$ sector graphs, denoted by $U_{1}, U_{2}, \cdots, U_{6^{5 t+4}}$, where these sector graphs is in the same and record as

$$
U_{j}=U\left(j \in\left\{1,2,3, \cdots, 6^{5 t+4}\right\}\right) .
$$

\section{THE EDGE-BALANCE INDEX SETS OF NESTED GRAPH

$$
C_{6^{m}} \times P_{m_{6}}(m \equiv 4(\bmod 4) 5)
$$

Lemma 1: For the power-cycle nested graph $C_{6^{m}} \times P_{m_{6}}$, when $m=4$

$$
\max \left\{E B I\left(C_{6^{4}} \times P_{4_{6}}\right)\right\}=1242
$$

Proof. There are 3102 edges in the power-cycle nested graph $C_{6^{4}} \times P_{4_{6}}$, let edge labeling function is an edgefriendly labeling that $|e(0)-e(1)| \leq 1$, there are 15510 edges.

In short, we only mark the 0 -edges of the graph, the left edges are 1-edges.

The edges on the second circle and the third circle are all 0 -edges except the edges $2_{3} 2_{4} 、 2_{9} 2_{10}$.

The edges linked to the vertices on the second circle $2_{i}(i=6 s+t, s=0,1, t=1,2, k=1,2 ; i=6 s+t, 3$ $\leq s \leq 5,3 \leq t \leq 5$ ) are all 0-edges,

The edges linked to the vertices on the third circle $3_{i}\left(i=6^{2} s+6 t+k-6 \mid s=0,1, t=1,2,1 \leq k \leq 6\right.$; $s=0,1, t=3,4,1 \leq k \leq 3 ; s=0,1, t=5,6, k=1,2$; $2 \leq s \leq 5,1 \leq t \leq 3,1 \leq k \leq 6 ; 2 \leq s \leq 5,4 \leq t \leq 6$, $k=1,2$;) are all 0-edges,

The 0-edges between the third circle and the forth circle

$3_{i} 4_{j}\left(i=6^{2} s+6 t+k-6, j=6(i-1)+l \mid s=0,1\right.$, $t=3,4,4 \leq k \leq 6, l=1,6 ; s=0,1, t=5,6,3 \leq k \leq$ $6, l=1,6 ; 2 \leq s \leq 5,4 \leq t \leq 6,3 \leq k \leq 6, l=1,6$;) are all 0 -edges,

The edges on the forth circle

$4_{i} 4_{j}\left(i=6^{3} s+6^{2} t+6 k+l-42, j=i+1 \mid s=0,1\right.$, $t=3,4,4 \leq k \leq 6, l=2,4 ; s=0,1, t=5,6,3 \leq k \leq$ $6, l=2,4 ; 2 \leq s \leq 5,4 \leq t \leq 6,3 \leq k \leq 6, l=2,4)$ are all 0-edges, then $v(0)=156,|v(0)-v(1)|=1242$. 
In this structure graph $C_{6^{4}} \times P_{4_{6}}$, it is apparent that the vertices are all saturated 0 -point and saturated 1-point. The degree of 0 -point is 9 , therefore, in order to change the label of one 0 -point, we need to interchange 50 edges. And it must increase the number of 0 -point and decrease the number of 1-point meanwhile. Then

$$
\max E B I\left(C_{6^{2}} \times P_{2_{6}}\right)=1242
$$

Lemma 2: For the single point sector sub-graph $U$, the maximum value of edge-balanced index is

$$
v(0)-v(1)=7464
$$

Proof. In order to conveniently research, in single point sector sub-graph $U$, there are 5 arcs, denoted as the first arc, the second arc, the third arc, the forth arc and the fifth arc from inside to outside in turn.

The starting point is denoted by $u$, the ray path meets the i-th arc at $1_{1}, 1_{2}, \cdots 1_{6} ; 2_{1}, 2_{2}, \cdots 2_{6^{2}} ; \cdots 5_{1}, 5_{2}, \cdots 5_{6^{5}}$ in clockwise order.

The ray path starts from $u$ as follows: $u \rightarrow 1_{i_{1}} \rightarrow 2_{i_{2}} \rightarrow 3_{i_{3}} \rightarrow 4_{i_{4}} \rightarrow 5_{i_{5}}\left(i_{k}=\left(i_{k-1}\right)+j ;\right.$ $\left.1 \leq i_{1}, j \leq 5\right)$

Now, we labeling sector graph $U$, the construction method of the 0 -edges is given, the rest are all labeled 0 edges.

The edges on the $i(1 \leq i \leq 4)$ arc are labeling 0 .

The edges linked to the vertices on the first arc $1_{i}(4 \leq i \leq 6)$ are all 0 -edges,

The edges linked to the vertices on the second arc $2_{i}(19 \leq i \leq 36)(i=6 s+t, 0 \leq s \leq 2, t=5,6)$ are all 0 edges,

The edges linked to the vertices on the third arc $3_{i}(109 \leq i \leq 216)\left(i=6^{2} s+6 t+k-6,0 \leq s \leq\right.$ $2,1 \leq t \leq 4, k=5,6,0 \leq s \leq 2 ; t=5,6,1 \leq k \leq 6$ are all 0-edges,

The edges linked to the vertices on the forth arc $4_{i}(649 \leq i \leq 930)\left(i=6^{3} s+6^{2} t+6 k+l-42 \mid 0 \leq\right.$ $s \leq 1,1 \leq t \leq 4,1 \leq k \leq 4, l=5,6 ; 0 \leq s \leq 1,1 \leq t \leq 4$, $k=5,6,1 \leq l \leq 6,0 \leq s \leq 1, t=5,6,1 \leq k \leq 6,1 \leq l$ $\leq 6)$ are all 0 -edges,

The 0-edges between the forth arc and the fifth arc $4_{i} 5_{j}\left(i=6^{3} s+6^{2} t+6 k+l-42, j=6(i-1)+p\right.$, $0 \leq s \leq 1,1 \leq t \leq 4,1 \leq k \leq 4,1 \leq l \leq 4, p=5,6)$ (931 $\leq i \leq 1296, j=5580+12 k+t, 0 \leq k \leq 182$, $t=1,12$ ) are all 0 -edges,

The edges on the fifth arc

$5_{i} 5_{j}\left(i=6^{4} s+6^{3} t+6^{2} k+6 l+p-258, j=i+1\right.$, $0 \leq s \leq 1,1 \leq t \leq 3,1 \leq k \leq 4,1 \leq l \leq 4, p=1,3 ; i$
$=5580+12 k+t, j=i+1,0 \leq k \leq 182, t=2,4$, $6,8,10)$ are all 0-edges.

By calculating, the sector graph $U$ is a friendly labeling, the vertices are all saturated 0-point and saturated 1-point. The degree of 0 -point is 9 , therefore, in order to change the label of one 0 -point, we need to interchange 50 -edges. And it must increase the number of 0 -point and decrease the number of 1-point, meanwhile. Then, the maximum value of edge-balanced index is $v(0)-v(1)=7464$.

Lemma 3: For the power-cycle nested graph $C_{6^{m}} \times P_{m_{6}}$, when $m \equiv 4(\bmod 5)$ and $m \geq 4$

$$
\begin{gathered}
\max \left\{\operatorname{EBI}\left(C_{6^{m}} \times P_{m_{6}}\right)\right\} \\
=\frac{5 \times 6^{m+4}+4 \times 6^{m+3}+3 \times 6^{m+2}+2 \times 6^{m+1}-16794}{6^{5}-1}
\end{gathered}
$$

Proof. When $m=4$, the formula can be proved by lemma 1.

Now, we prove the formula is established when $m \geq 4$.

First of all, structure the graph of maximum edgebalanced index.

When $m \geq 4$,

$$
C_{6^{\mathrm{m}}} \times P_{m_{6}}=C_{6^{4}} \times P_{4_{6}} \cup\left(\bigcup_{i=0}^{\frac{m-4}{5}} V_{i}\right),
$$

number the foundation graph and the nested-cycle subgraph.

The label of the foundation graph $C_{6^{m}} \times P_{m_{6}}$ is the same as lemma 1.

The label of the nested-cycle sub-graph is the same as lemma 2.In the power-cycle nested graph $C_{6^{m}} \times P_{m_{6}}$, the foundation graph has the maximum radix number of edge-balanced index.

$u$ is an undefined point in each of the single point sector sub-graph that won't affect the global maximum radix number of edge-balanced index. The nested-cycle sub-graph has the maximum radix number of edgebalanced index, then $\max \{E B I(U)\}=7464$

In power-cycle nested graph $\mathrm{h} C_{6^{m}} \times P_{m_{6}}$, the first nested-cycle has $6^{4}$ single point sector sub-graph, the second nested-cycle has $6^{9}$ single point sector subgraph $\cdots$, the $i$ nested-cycle has $6^{m-5}$ single point sector sub-graph, then in the power-cycle nested graph $C_{6^{m}} \times P_{m_{6}}$

$$
\begin{aligned}
& |v(0)-v(1)|=\left(6^{4}+6^{9}+\cdots 6^{m-10}+6^{m-5}\right) \times 7464+1242= \\
& \frac{5 \times 6^{m+4}+4 \times 6^{m+3}+3 \times 6^{m+2}+2 \times 6^{m+1}-16794}{6^{5}-1}
\end{aligned}
$$

Therefore, in power-cycle nested graph $\mathrm{h}_{6^{m}} \times P_{m_{6}}$

$$
\max \left\{\operatorname{EBI}\left(C_{6^{m}} \times P_{m_{6}}\right)\right\}=
$$




$$
\frac{5 \times 6^{m+4}+4 \times 6^{m+3}+3 \times 6^{m+2}+2 \times 6^{m+1}-16794}{6^{5}-1}
$$

In the following proof, make the e labeled graph that its index is

$$
\begin{gathered}
\max \left\{E B I\left(C_{6^{m}} \times P_{m_{6}}\right)\right\} \\
=\frac{5 \times 6^{m+4}+4 \times 6^{m+3}+3 \times 6^{m+2}+2 \times 6^{m+1}-16794}{6^{5}-1}
\end{gathered}
$$

In the Lemma 3 as the foundation graph and transform it to be the graph corresponding all index .

Obviously, the label of the sub-graph is the same as original graph.

Lemma 4 For the single point sector sub-graph $U$, $\{7462,7460,7458 \cdots 6,4,2,0\} \subset E B I(U)$.

Proof. In sector graph $U$ from lemma 2, there is $|v(0)-v(1)|=7464$, interchange the 0-edges and 1edges partly and the following steps are all on the basis of the previous labeled graph. We use $i_{g} j_{h} \leftrightarrow s_{t} p_{l}$ to present that the edge $i_{g} j_{h}$ is from 0-edge into 1-edge and edge $s_{t} p_{l}$ is from1-edge into 0 -edge.

Step1:

$$
\begin{gathered}
4_{i} 5_{j} \leftrightarrow 5_{j-1} 5_{j}\left(i=6^{3} s+6^{2} t+6 k+l-6^{2}-6,\right. \\
j=6(i-1)+p, s=0,1,2, t=1,2,3, k=1,2,3,4, \\
l=1,2,3,4, p=5,6) \\
|v(1)-v(0)|=\{7462,7460, \cdots, 6698,6696\}
\end{gathered}
$$

Step2:

$$
\begin{gathered}
4_{i} 5_{j} \leftrightarrow 5_{j-1} 5_{j}\left(i=6^{3} s+6^{2} t+6 k+l-6^{2}-6,\right. \\
j=6(i-1)+p, s=0,1,2, t=1,2,3, k=1,2,3 \\
, 4, l=5,6, p=2,4,6) \\
|v(1)-v(0)|=\{6694,6692, \cdots, 5930,5928\}
\end{gathered}
$$

Step3:

$$
\begin{gathered}
4_{i} 5_{j} \leftrightarrow 5_{j-1} 5_{j}\left(i=6^{3} s+6^{2} t+6 k+l-6^{2}-6,\right. \\
j=6(i-1)+p, s=0,1,2, t=1,2,3,4, k=5,6, \\
l=1,2,3,4,5,6, p=2,4,6) \\
|v(1)-v(0)|=\{5926,5924, \cdots, 4478,4476\}
\end{gathered}
$$

Step4:

$$
\begin{gathered}
4_{i} 5_{j} \leftrightarrow 5_{j-1} 5_{j}\left(i=6^{3} s+6^{2} t+6 k+l-6^{2}-6,\right. \\
j=6(i-1)+p, s=0,1,2, t=5,6, k=1,2,3,4,
\end{gathered}
$$

$$
\begin{gathered}
5,6, l=1,2,3,4,5,6, p=2,4,6) \\
|v(1)-v(0)|=\{4774,4772, \cdots, 3050,3048\}
\end{gathered}
$$

Step5:

$$
\begin{aligned}
4_{i} 5_{j} \leftrightarrow 5_{j-1} 5_{j}( & 649 \leq i \leq 930, j=6(i-1) \\
& +k, k=2,4,6) \\
|v(1)-v(0)| & =\{3046,3044, \cdots, 794,792\}
\end{aligned}
$$

Step6:

$$
\begin{gathered}
4_{i} 5_{j} \leftrightarrow 5_{j+1} 5_{j}(931 \leq i \leq 1296, j=5580 \\
+12 k+1,0 \leq k \leq 182) \\
4_{i} 5_{j} \leftrightarrow 5_{j-1} 5_{j}(931 \leq i \leq 1296, j=5580+12 k \\
+12,0 \leq k \leq 182) \\
|v(1)-v(0)|=\{790,788, \cdots, 62,60\}
\end{gathered}
$$

Step7:

$$
\begin{aligned}
& 4_{i} 5_{j} \leftrightarrow 5_{j-1} 5_{j} \quad(1267 \leq i \leq 1296, j=7596 \\
&+12 k+p, 0 \leq k \leq 14, p=3,5,9,12) \\
&|v(1)-v(0)|=\{58,56, \cdots, 4,2,0\}
\end{aligned}
$$

Proposition is proved.

Theorem 1: For the power-cycle nested graph $C_{6^{m}} \times P_{m_{6}}$, when $m \equiv 4(\bmod 5)$ and $m \geq 4$

$$
\begin{gathered}
\frac{5 \times 6^{m+4}+4 \times 6^{m+3}+3 \times 6^{m+2}+2 \times 6^{m+1}-16794}{6^{5}-1} \\
-2, \cdots 4,3,2,1,0\} \subset E B I\left(C_{6^{m}} \times P_{m_{6}}\right) .
\end{gathered}
$$

Proof. In the following proof, every step is all on the basis of the previous labeled graph we will make the labeled graph that its index is

$$
\begin{gathered}
\max \left\{\operatorname{EBI}\left(C_{6^{m}} \times P_{m_{6}}\right)\right\} \\
=\frac{5 \times 6^{m+4}+4 \times 6^{m+3}+3 \times 6^{m+2}+2 \times 6^{m+1}-16794}{6^{5}-1}
\end{gathered}
$$

in the Lemma 2 as the foundation graph and transform it to be the graph corresponding all index.

(1) The proof about even index.

Make the following transformation

Step1:

$3_{i} 4_{j} \leftrightarrow 4_{j-1} 4_{j}\left(i=6^{2} s+6 t+k-6, j=6(i-1)\right.$ 
$+l \mid s=0,1, t=1,2,1 \leq k \leq 6, l=2,4,6, s=0,1$, $3 \leq t \leq 6, k=1,2, l=2,4,6 ; 2 \leq s \leq 5,1 \leq t \leq 3$, $1 \leq k \leq 6, l=2,4,6 ; 2 \leq s \leq 5,4 \leq t \leq 6, k=1,2$,

$$
l=2,4,6)
$$

Step2:

$$
\begin{gathered}
3_{i} 4_{j} \leftrightarrow 4_{j-1} 4_{j}\left(i=6^{2} s+6 t+k-6, j=6(i-1)\right. \\
+l \mid s=0,1,3 \leq t \leq 6,4 \leq k \leq 6, l=6 ; 2 \leq s \leq \\
5,4 \leq t \leq 6,3 \leq k \leq 6, l=6)
\end{gathered}
$$

Step 3:

$$
\begin{gathered}
3_{i} 4_{j} \leftrightarrow 4_{j+1} 4_{j}\left(i=6^{2} s+6 t+k-6, j=6(i-1)\right. \\
+l, s=0,1,3 \leq t \leq 6,4 \leq k \leq 6, l=1 ; 2 \leq s \leq \\
5,4 \leq t \leq 6,3 \leq k \leq 6, l=1)
\end{gathered}
$$

Step 4:

$$
\begin{aligned}
& 34_{i} \leftrightarrow 4_{j-1} 4_{j}\left(i=6^{2} s+6 t+k-6, j=6(i-1)\right. \\
& +l \mid 2 \leq s \leq 4,1 \leq t \leq 3,1 \leq k \leq 6, l=3 ; s=5 \\
& t=1,2,1 \leq k \leq 6, l=3 ; s=5, t=3,1 \leq k \leq 3, l=3)
\end{aligned}
$$

Then obtain following index respectively

$$
\begin{gathered}
\left\{\frac{5 \times 6^{m+4}+4 \times 6^{m+3}+3 \times 6^{m+2}+2 \times 6^{m+1}-16794}{6^{5}-1}-2,\right. \\
\frac{5 \times 6^{m+4}+4 \times 6^{m+3}+3 \times 6^{m+2}+2 \times 6^{m+1}-16794}{6^{5}-1} \\
-4, \cdots, \\
\frac{5 \times 6^{m+4}+4 \times 6^{m+3}+3 \times 6^{m+2}+2 \times 6^{m+1}-16794}{6^{5}-1} \\
-1242\}
\end{gathered}
$$

Then make the transformation from step one to step seven in lemma 3 for sector graph $U_{1}, U_{2}, \cdots, U_{6^{36}}$ which belong to $V_{0}$ in turn and obtain the following index respectively.

$$
\begin{gathered}
\left\{\frac{5 \times 6^{m+4}+4 \times 6^{m+3}+3 \times 6^{m+2}+2 \times 6^{m+1}-16794}{6^{5}-1}-1244,\right. \\
\frac{5 \times 6^{m+4}+4 \times 6^{m+3}+3 \times 6^{m+2}+2 \times 6^{m+1}-16794}{6^{5}-1}-1246, \cdots, \\
\frac{5 \times 6^{m+4}+4 \times 6^{m+3}+3 \times 6^{m+2}+2 \times 6^{m+1}-16794}{6^{5}-1} \\
\left.-6^{4} \times 7464-1242\right\}
\end{gathered}
$$

Finally make the transformation for $V_{1} V_{2} \cdots V_{t}$ in turn, and then obtain following index respectively

$$
\begin{gathered}
\left(\frac{5 \times 6^{m+4}+4 \times 6^{m+3}+3 \times 6^{m+2}+2 \times 6^{m+1}-16794}{6^{5}-1}-6^{4} \times 7464-1242,\right. \\
\frac{5 \times 6^{m+4}+4 \times 6^{m+3}+3 \times 6^{m+2}+2 \times 6^{m+1}-16794}{6^{5}-1} \\
\left.-6^{4} \times 7464-1244, \cdots 4,2,0\right\}
\end{gathered}
$$

(2) The proof about odd index.

First make the transformation $1_{1} 2_{3} \leftrightarrow 2_{2} 2_{3}$

Then make the following transformation

Step 1:

$$
\begin{gathered}
3_{i} 4_{j} \leftrightarrow 4_{j-1} 4_{j}\left(i=6^{2} s+6 t+k-6, j=6(i-1)\right. \\
+l \mid s=0,1, t=1,2,1 \leq k \leq 6, l=2,4,6, s=0, \\
1,3 \leq t \leq 6, k=1,2, l=2,4,6 ; 2 \leq s \leq 5,1 \leq t \leq \\
3,1 \leq k \leq 6, l=2,4,6 ; 2 \leq s \leq 5,4 \leq t \leq 6, k= \\
1,2, l=2,4,6)
\end{gathered}
$$

Step2:

$$
\begin{gathered}
3_{i} 4_{j} \leftrightarrow 4_{j-1} 4_{j}\left(i=6^{2} s+6 t+k-6, j=6(i-1)\right. \\
+l \mid s=0,1,3 \leq t \leq 6,4 \leq k \leq 6, l=6 ; 2 \leq s \leq 5, \\
4 \leq t \leq 6,3 \leq k \leq 6, l=6)
\end{gathered}
$$

Step3:

$$
\begin{gathered}
3_{i} 4_{j} \leftrightarrow 4_{j+1} 4_{j}\left(i=6^{2} s+6 t+k-6, j=6(i-1)\right. \\
+l, s=0,1,3 \leq t \leq 6,4 \leq k \leq 6, l=1 ; 2 \leq s \leq 5, \\
4 \leq t \leq 6,3 \leq k \leq 6, l=1)
\end{gathered}
$$

Step4:

$3_{i} 4_{j} \leftrightarrow 4_{j-1} 4_{j}\left(i=6^{2} s+6 t+k-6, j=6(i-1)\right.$ $+l \mid 2 \leq s \leq 4,1 \leq t \leq 3,1 \leq k \leq 6, l=3 ; s=5, t=$ $1,2,1 \leq k \leq 6, l=3 ; s=5, t=3, k=1,2, l=3$ )

Then obtain following index respectively

$$
\begin{aligned}
& \left\{\frac{5 \times 6^{m+4}+4 \times 6^{m+3}+3 \times 6^{m+2}+2 \times 6^{m+1}-16794}{6^{5}-1}-1,\right. \\
& \frac{5 \times 6^{m+4}+4 \times 6^{m+3}+3 \times 6^{m+2}+2 \times 6^{m+1}-16794}{6^{5}-1}-3, \cdots, \\
& \left.\frac{5 \times 6^{m+4}+4 \times 6^{m+3}+3 \times 6^{m+2}+2 \times 6^{m+1}-16794}{6^{5}-1}-1241\right\}
\end{aligned}
$$


Then make the transformation from step one to step seven in lemma 3 for sector graph $U_{1}, U_{2}, \cdots, U_{6^{36}}$ which belong to $V_{0}$ in turn and obtain the following index respectively.

$$
\begin{gathered}
\left\{\frac{5 \times 6^{m+4}+4 \times 6^{m+3}+3 \times 6^{m+2}+2 \times 6^{m+1}-16794}{6^{5}-1}-1243,\right. \\
\frac{5 \times 6^{m+4}+4 \times 6^{m+3}+3 \times 6^{m+2}+2 \times 6^{m+1}-16794}{6^{5}-1}-1245, \cdots, \\
\frac{5 \times 6^{m+4}+4 \times 6^{m+3}+3 \times 6^{m+2}+2 \times 6^{m+1}-16794}{6^{5}-1} \\
\left.-6^{4} \times 7464-1241\right\}
\end{gathered}
$$

Finally make the transformation for $V_{1} V_{2} \cdots V_{t}$ in turn, and then obtain following index respectively

$$
\begin{aligned}
& \left\{\frac{5 \times 6^{m+4}+4 \times 6^{m+3}+3 \times 6^{m+2}+2 \times 6^{m+1}-16794}{6^{5}-1}\right. \\
& -6^{4} \times 7464-1243, \\
& \frac{5 \times 6^{m+4}+4 \times 6^{m+3}+3 \times 6^{m+2}+2 \times 6^{m+1}-16794}{6^{5}-1} \\
& \left.-6^{4} \times 7464-1245, \cdots 5,3,1\right\}
\end{aligned}
$$

In conclusion, theorem was proved.

Based on Lemma 3and Theorem 1, we have

\section{CONCLUSION}

For the power-cycle nested graph $C_{6^{m}} \times P_{m_{6}}$, when $m \equiv 4(\bmod 5)$ and $m \geq 4$

$$
\begin{gathered}
\left\{\frac{5 \times 6^{m+4}+4 \times 6^{m+3}+3 \times 6^{m+2}+2 \times 6^{m+1}-16794}{6^{5}-1},\right. \\
\frac{5 \times 6^{m+4}+4 \times 6^{m+3}+3 \times 6^{m+2}+2 \times 6^{m+1}-16794}{6^{5}-1} \\
-1, \cdots 4,3,2,1,0\} \subset E B I\left(C_{6^{m}} \times P_{m_{6}}\right)
\end{gathered}
$$

The theory of this thesis can be applied to computer science and information engineering and the proving method can be a reference to solve the problem of the power-cycle nested graph $C_{6^{m}} \times P_{m_{6}}$.

\section{ACKNOWLEDGMENT}

Thanks for project supported by the Innovating Foundation of Henan Polytechnic University for the Master Thesis. Thanks for project supported by Applied
Mathematics Provincial-level Key Discipline of Henan Province and Operational Research and Cybernetics Key Discipline of Henan Polytechnic University. Thanks for project supported by the Ph.D. Foundation of Henan Polytechnic University Grant No.B2011-085.

\section{REFERENCES}

[1] M.C.Kong, Sin-Min Lee, On Edge-Balanced Graphs[J],Graph Theory,Combinatori and C Algorithms, V.1,711 -722(1995).

[2] B.L.Chen, K.C. Huang and Shi-Shen Liu,On edge-balanced multigraphs,Journal of Combinatorial Mathematics and Combinatorial Computing,42 (2002),177-185.R. Ni cole.

[3] Harris Kwong and H.K. Ng, On Friendly Index Sets of 2-regular graphs, iscrete Mathematics. 308(2008), 5522-5532.

[4] Ebrahim Salehi and Sin-Min Lee, Friendly index sets of trees, Congressus Numerantium 178 (2006), pp. 173-183.

[5] Suh-Ryung Kim, Sin-Min Lee and Ho Kuen Ng, On Balancedness of Some Graph Constructions, Journal of Combinatorial Mathematics and Combinatorial Computing, 66 (2008) 3-16.

[6] Alexander Nien-Tsu Lee, Sin-Min Lee and Ho Kuen Ng, On The Balance Index Set of Graphs, Journal of Combinatorial Mathematics and Combinatorial Computing., 66 (2008), 133-150.

[7] D.Chopra , H. H. Su ,Sin-Min Lee,On edgebalance index sets of wheels, Int. J. Contemp. Math. Sciences, Vol. 5, 2010, no. 53, 2605 2620.

[8] Sin-Min Lee, C.C.Chou, M.Galiardi, M.Kong, D.Perry, H.H. Su, On edge-balance index sets of L-product of cycles with stars, Part 1, JCMCC 78(2011),195-211.

[9] Juan Lu, Yu-ge Zheng. On the edge-balance index sets of $\mathrm{B}(\mathrm{n})$, proceedings of the Jang-jeon Mathemati- cal Society,Vol.12 NO.1 June (2009),37-44.

[10] Ji Yurong, Yuge Zheng. On edge-balance index sets of the complete graphs, ICIME 2010-2010 2nd IEEE International Conference on Information Management and Engineering, v6, $\mathrm{p}$ 309-311, 2010.

[11] Yuge Zheng Jingjing Yao: The Edge-balance Index Sets of Nested Graph with the Unlimited Pathes and Equal Pathes and Circle(1), Journal of Shanghai Jiaotong University, 47(7)(2013) (Articles In Press.

Qingwen Zhang: Student for Master degree for Applied Mathematics in Henan Polytechnic University, major in Graph theory and combinatorial mathematics.

Yuge Zheng: Professor of Henan Polytechnic University, interested in Graph theory and combinatorial mathematics. 